\title{
Aero-Thermal Calibration of the NASA Glenn Icing Research Tunnel (2012 Test)
}

A major modification of the refrigeration plant and heat exchanger at the NASA Glenn Icing Research Tunnel (IRT) occurred in autumn of 2011. It is standard practice at NASA Glenn to perform a full aero-thermal calibration of the test section of a wind tunnel facility upon completion of major modifications. This paper will discuss the tools and techniques used to complete an aero-thermal calibration of the IRT and the results that were acquired. The goal of this test entry was to complete a flow quality survey and aero-thermal calibration measurements in the test section of the IRT. Test hardware that was used includes the 2D Resistive Temperature Detector (RTD) array, 9-ft pressure survey rake, hot wire survey rake, and the quick check survey rake. This test hardware provides a map of the velocity, Mach number, total and static pressure, total temperature, flow angle and turbulence intensity. The data acquired were then reduced to examine pressure, temperature, velocity, flow angle, and turbulence intensity. Reduced data has been evaluated to assess how the facility meets flow quality goals. No icing conditions were tested as part of the aero-thermal calibration. However, the effects of the spray bar air injections on the flow quality and aero-thermal calibration measurements were examined as part of this calibration. 


\section{IRT 2011-12 Cooling System Upgrade}

\section{Aero-Thermal Calibration of the NASA Glenn Icing Research Tunnel (2012 Test)}

Christine Pastor-Barsi / Sierra Lobo, Inc.

E. Allen Arrington / Sierra Lobo, Inc Judith Foss Van Zante, Ph.D. / Sierra Lobo, Inc

$4^{\text {th }}$ Atmospheric and Space Environments Conference June 26, 2012 


\section{Session Summary}

\begin{tabular}{|l|l|l|}
\hline Time & Topic & Presenter \\
\hline $0800-0900$ & IRT Upgrade and Cloud Cal & Van Zante / NASA-SLI \\
\hline $0900-0930$ & IRT Test Section Aero-Thermal Cal & $\begin{array}{l}\text { Pastor-Barsi / NASA- } \\
\text { SLI }\end{array}$ \\
\hline $0930-1000$ & IRT Plenum Aero-Thermal Cal & Steen / NASA-SLI \\
\hline $1000-1030$ & $\begin{array}{l}\text { VIRT: Air Flow and Liquid Water } \\
\text { Concentration Simulations }\end{array}$ & Clark / UVa \\
\hline $1030-1100$ & $\begin{array}{l}\text { VIRT: Drop Concentration and Flux } \\
\text { on Aerodynamic Surfaces }\end{array}$ & Triphahn / UIUC \\
\hline $1100-1130$ & 3D Laser Scanner in IRT & Lee / NASA-ASRC \\
\hline
\end{tabular}




\section{Why Aero-Thermal?}

- Calibration and Acceptance of Icing Wind Tunnels (Aerospace Recommended Practice 5905)

\begin{tabular}{|r|c|c|c|}
\hline Parameter & $\begin{array}{c}\text { Measurement } \\
\text { Uncertainty }\end{array}$ & $\begin{array}{c}\text { Test Section Spatial } \\
\text { Uniformity }\end{array}$ & $\begin{array}{c}\text { Tunnel Centerline } \\
\text { Temporal Stability }\end{array}$ \\
\hline Airspeed & $\pm 1 \%$ & $\pm 2 \%$ & $\pm 2 \%$ \\
Static air temperature, -30 through $+5{ }^{\circ} \mathrm{C}$ & $\pm 2{ }^{\circ} \mathrm{C}$ & $\pm 1{ }^{\circ} \mathrm{C}$ & $\pm 0.5^{\circ} \mathrm{C}$ \\
Flow Angularity & $\pm 0.25^{\circ}$ & \pm 2 & $\mathrm{~N} / \mathrm{A}$ \\
Turbulence $\left(P_{\text {air }}=0\right.$ psig) & $\pm 0.25 \%$ & $<2 \%$ & $\pm 2 \%$ \\
Turbulence $\left(P_{\text {air }}=60\right.$ psig) & $\pm 0.25 \%$ & $<2 \%$ & $\pm 2 \%$ \\
\hline
\end{tabular}

- AIAA Recommended Practice (AIAA R-0932003) Calibration of Subsonic and Transonic Wind Tunnels

- Health monitoring

Goal - Calibration curve 


\section{Outline}

- 2012 Aero-thermal testing occurred January and May 2012 after major facility upgrade

- Previous full Calibration January 2009

- Hardware

- 9-ft survey rake

- 2D RTD array

- Hot wire/film survey rake

- Results

- Summary 


\section{Icing Research Tunnel}

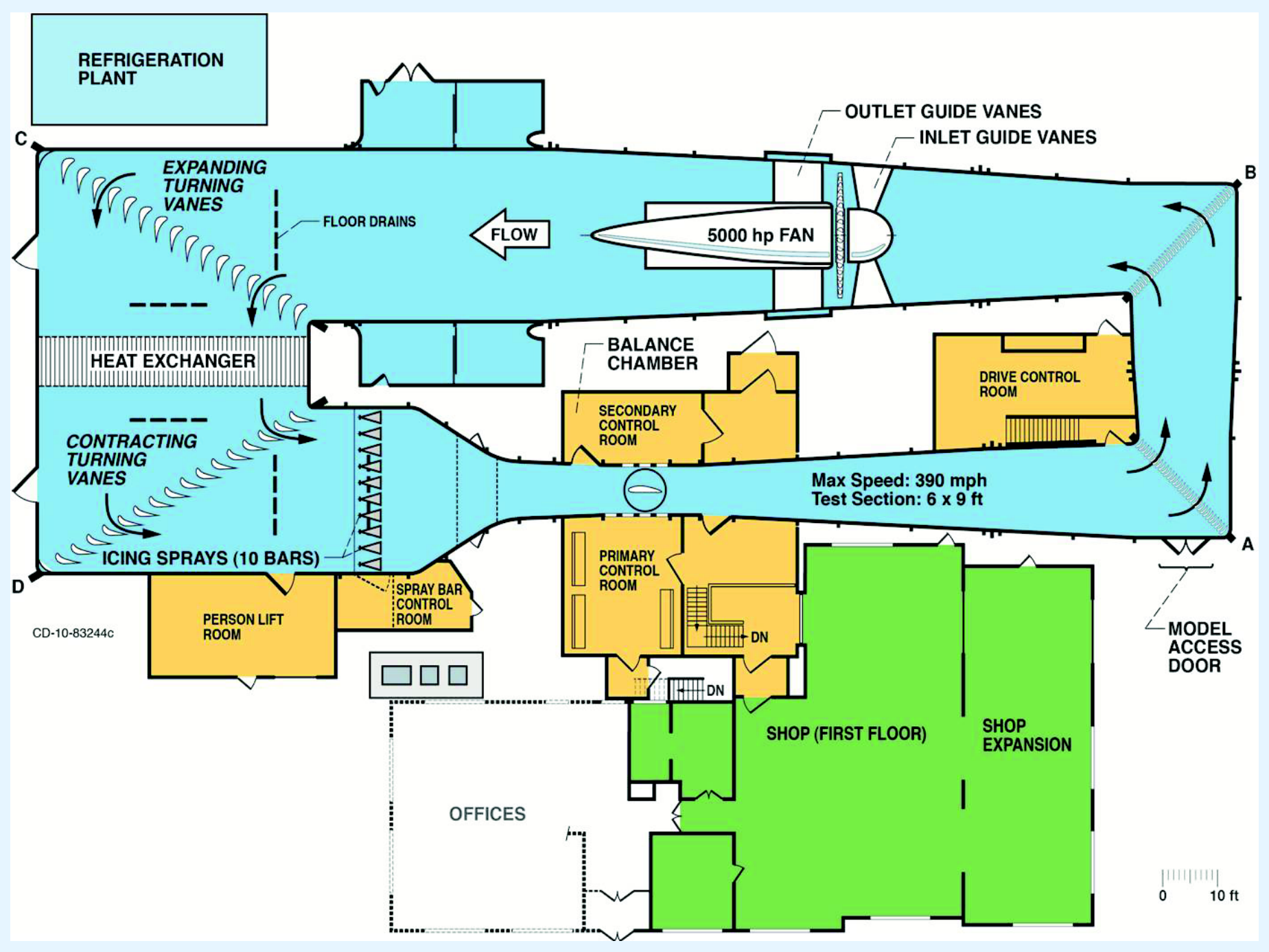




\section{D RTD Array}

-7x7 grid of RTDs (49 total)

-Probes are 4-wire RTDs with a ceramic capsule sensor

- Temperature flow recovery calibration completed in 2005 (AIAA 2005-4276)

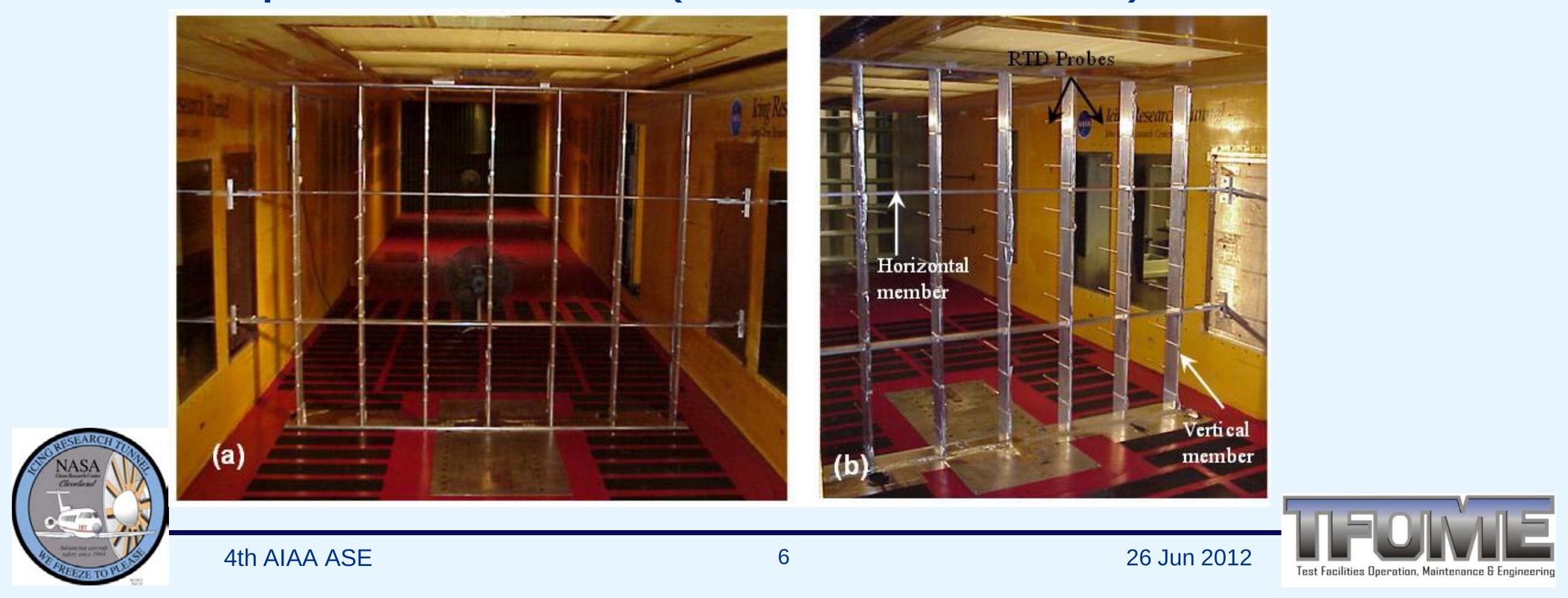




\section{Test Matrix 2D RTD Array}

\begin{tabular}{|c|c|c|}
\hline $\begin{array}{c}\text { Test Section total } \\
\text { temperature, } T_{T, t s}{ }^{\circ} \mathrm{C}\end{array}$ & $\begin{array}{c}\text { Test Section } \\
\text { Airspeed, } U_{\text {ts }} \text { knots }\end{array}$ & $\begin{array}{c}\text { Spraybar air pressure, } \\
P_{\text {air }}, \text { psig }\end{array}$ \\
\hline-30 & $50,130,220,300$ & $0,30,60$ \\
-20 & $50,130,220,300$ & $0,30,60$ \\
-10 & $50,130,220,300$ & $0,30,60$ \\
-3 & $50,130,220,300$ & $0,30,60$ \\
-2 & $50,130,220,300$ & $0,30,60$ \\
-1 & $50,130,220,300$ & $0,30,60$ \\
0 & $50,130,220,300$ & $0,30,60$ \\
1 & $50,130,220,300$ & $0,30,60$ \\
2 & $50,130,220,300$ & $0,30,60$ \\
3 & $50,130,220,300$ & $0,30,60$ \\
5 & $50,130,220,300$ & $0,30,60$ \\
\hline
\end{tabular}




\section{9-ft Survey Rake}

-Positioning every 6inches vertically

-11 Hemisphericalhead 5-hole pressure probes

-Measures total and static pressures

-Probes calibrated in


1996 (AIAA 96-2201) 


\section{Test Matrix 9-ft Survey Rake}

\begin{tabular}{|r|c|c|c|}
\hline $\begin{array}{r}\text { Rake Position } \\
\text { (horizontal) }\end{array}$ & $\begin{array}{c}\text { Static } \\
\text { temperature, } T_{T, t s}{ }^{\circ} \mathrm{C}\end{array}$ & $\begin{array}{c}\text { Test Section } \\
\text { Airspeed, } U_{\text {ts }} \text { knots }\end{array}$ & $\begin{array}{c}\text { Spraybar air pressure, } \\
P_{\text {air }}, \text { psig }\end{array}$ \\
\hline CL & 4.0 & $50,90,120,150,170,200,250$ & $0,30,60$ \\
CL-6" & 4.0 & $50,90,120,150,170,200,250$ & $0,30,60$ \\
CL+6" & 4.0 & $50,90,120,150,170,200,250$ & $0,30,60$ \\
CL-12" & 4.0 & $50,90,120,150,170,200,250$ & $0,30,60$ \\
CL+12" & 4.0 & $50,90,120,150,170,200,250$ & $0,30,60$ \\
CL-18' & 4.0 & $50,90,120,150,170,200,250$ & $0,30,60$ \\
CL+18' & 4.0 & $50,90,120,150,170,200,250$ & $0,30,60$ \\
CL-24" & 4.0 & $50,90,120,150,170,200,250$ & $0,30,60$ \\
CL+24 & 4.0 & $50,90,120,150,170,200,250$ & $0,30,60$ \\
\hline
\end{tabular}




\section{Hot Wire Survey Rake}

- Hot wire survey has 5 positions across the test section in 18-inch increments

-Testing is done in only the incompressible regime, $M<0.3$ (180 knots)

-Calibrations of the hot wires are performed in situ

- Only single wires/films normal to the axial velocity are

\section{used}

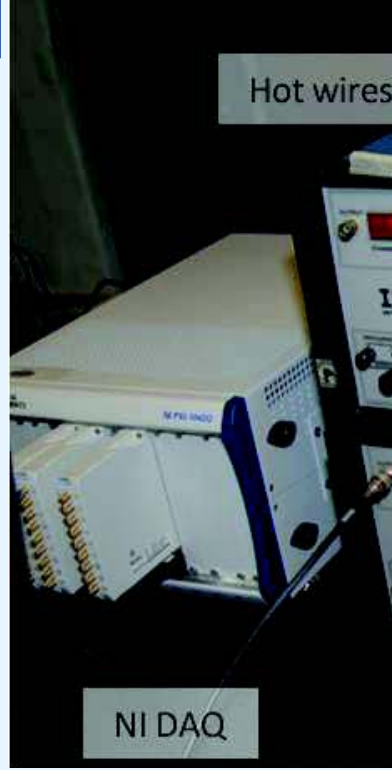

NI DAQ

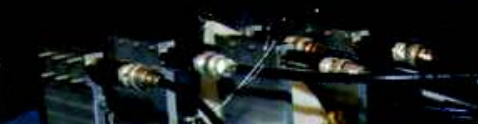

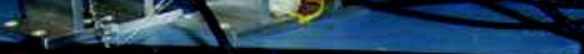

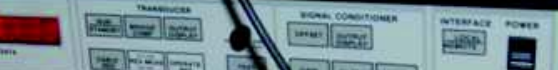
FA.100
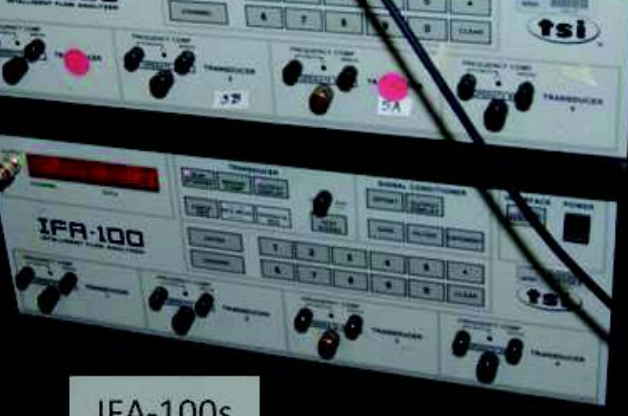

IFA-100s

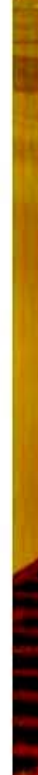

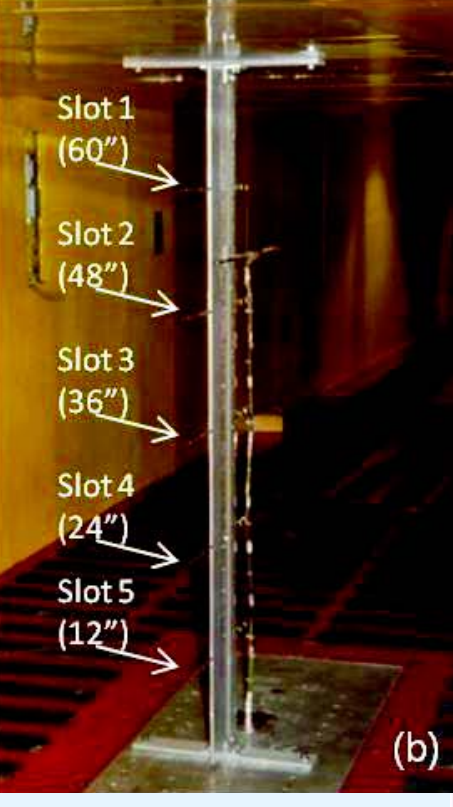




\section{Test Matrix Hot Wire Survey Rake}

\begin{tabular}{|r|c|c|c|}
\hline $\begin{array}{r}\text { Rake Position } \\
\text { (lateral) }\end{array}$ & $\begin{array}{c}\text { Test Section total } \\
\text { temperature, } T_{T, t s}{ }^{\circ} \mathrm{C}\end{array}$ & $\begin{array}{c}\text { Test Section } \\
\text { Airspeed, } U_{t s} \text { knots }\end{array}$ & $\begin{array}{c}\text { Spraybar air pressure, } \\
P_{\text {air }}, \text { psig }\end{array}$ \\
\hline CL & 7.8 & $50,90,120,150,170$ & $0,30,60$ \\
CL-18" & 7.8 & $50,90,120,150,170$ & $0,30,60$ \\
CL-36" & 7.8 & $50,90,120,150,170$ & $0,30,60$ \\
CL+18" & 7.8 & $50,90,120,150,170$ & $0,30,60$ \\
CL+36" & 7.8 & $50,90,120,150,170$ & $0,30,60$ \\
\hline
\end{tabular}




\section{Facility Instrumentation}

- D-corner RTD Array

- 24-probe array, 4 rows of 6 probes

- North and South Bellmouth Pitot-Static probes

- Measure total and delta pressure

- Heated to prevent icing 


\section{Mach number}

\section{Target Test}

Conditions:

150 knots,

$\mathrm{TSDC}=4.0^{\circ} \mathrm{C}$
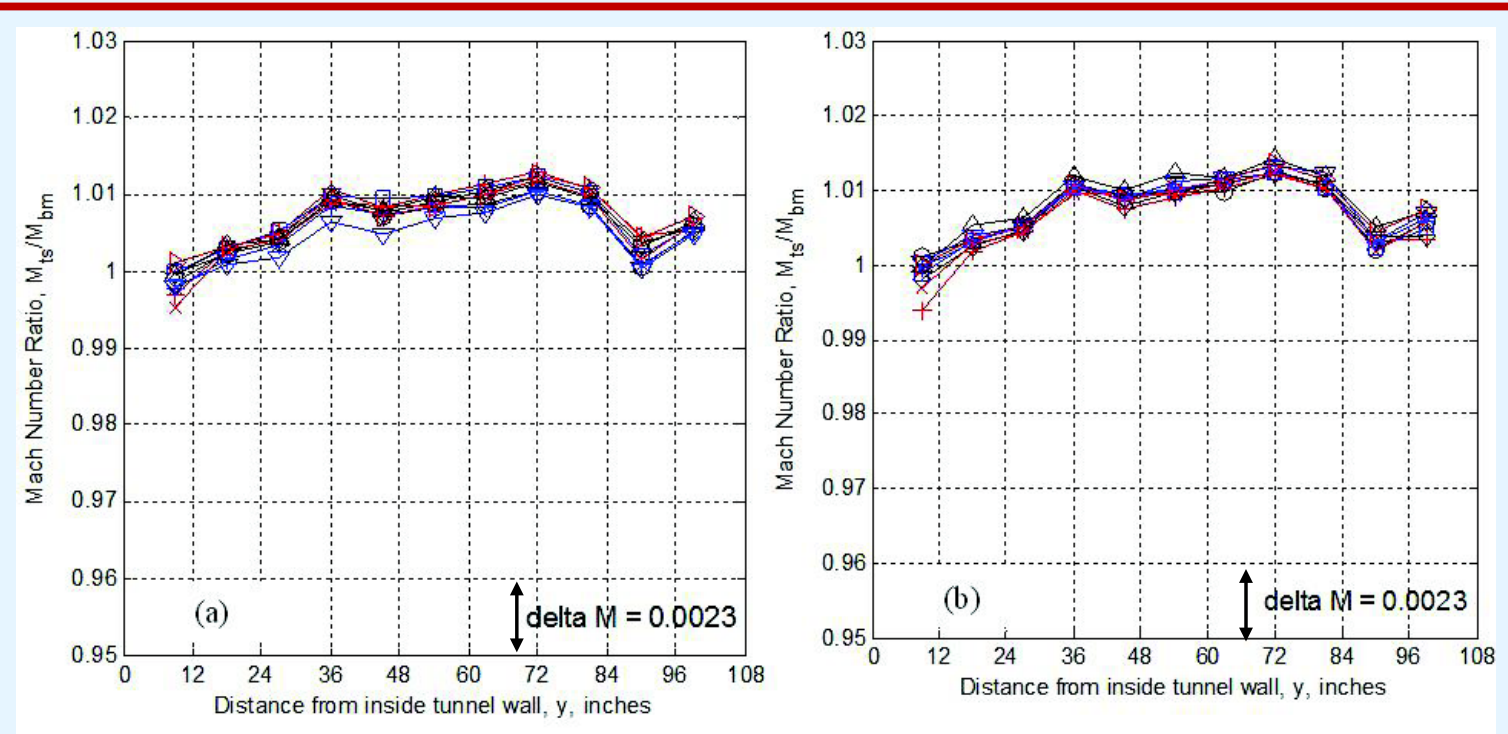

(a) 0 psig

(b) 30 psig

(c) 60 psig

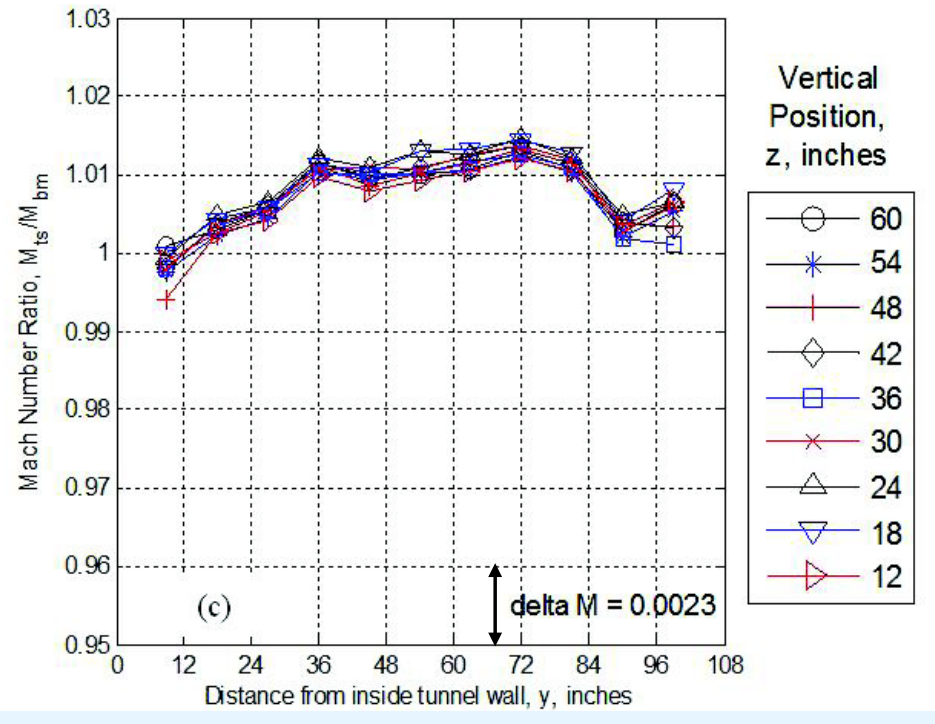




\section{Total Pressure}

\section{Target Test} Conditions: 150 knots, $\mathrm{TSDC}=4.0^{\circ} \mathrm{C}$
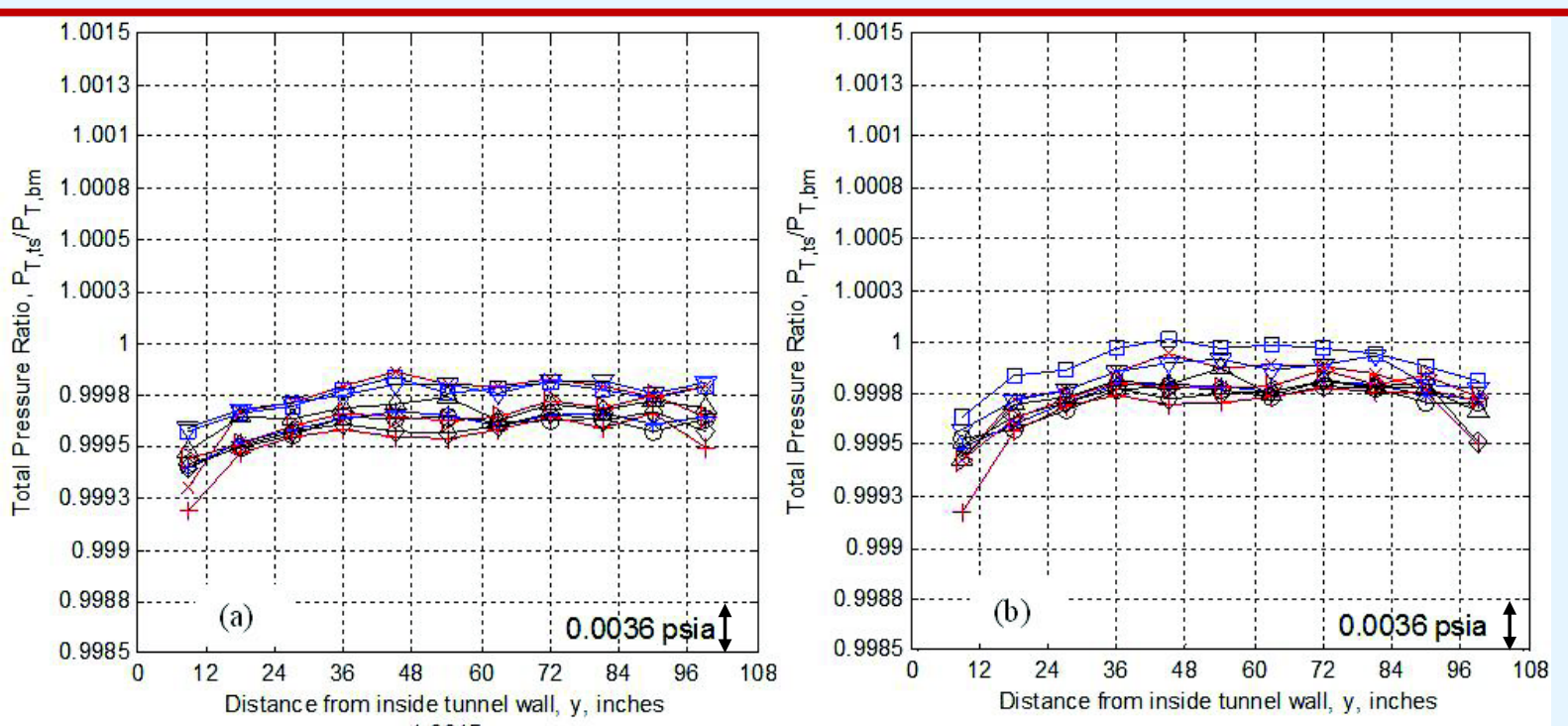

(a) 0 psig

(b) 30 psig

(c) 60 psig

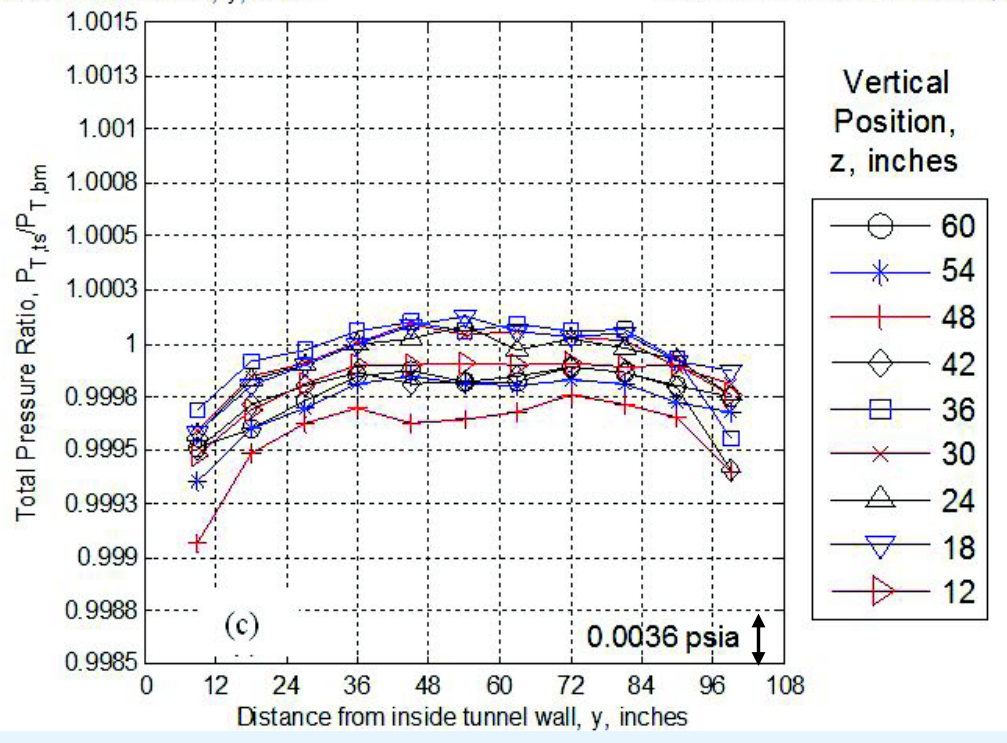




\section{Static Pressure}

\section{Target Test} Conditions: 150 knots, $\mathrm{TSDC}=4.0^{\circ} \mathrm{C}$
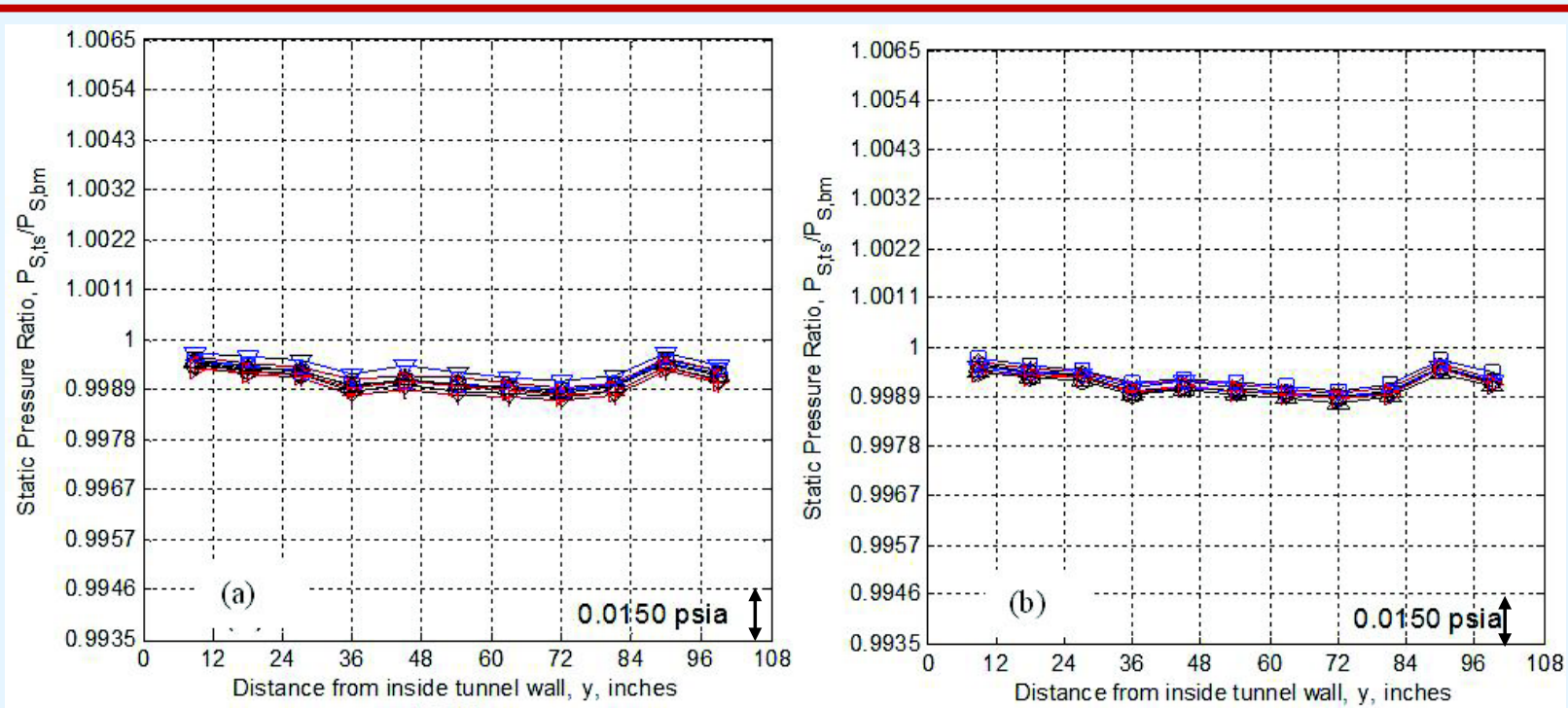

(a) 0 psig

(b) 30 psig

(c) 60 psig

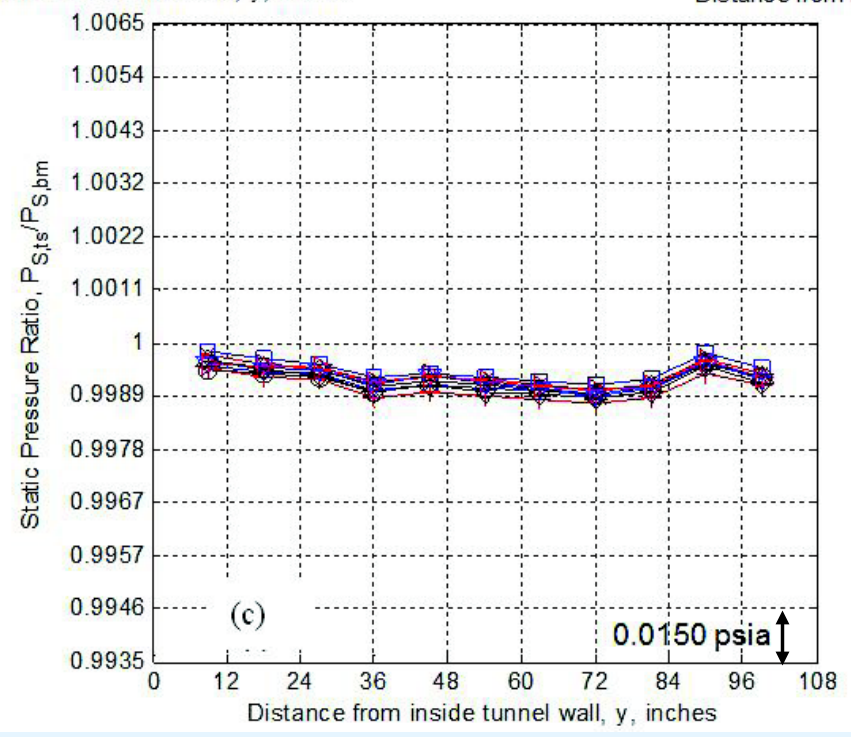

Vertical

Position,

$z$, inches

\begin{tabular}{|c|}
\hline \multirow{2}{*}{$\begin{array}{r}0 \\
* 54 \\
\end{array}$} \\
\hline \\
\hline$-1-48$ \\
\hline$>42$ \\
\hline$\square-36$ \\
\hline$\longrightarrow-30$ \\
\hline$\triangle-24$ \\
\hline$\nabla-18$ \\
\hline$\longrightarrow-12$ \\
\hline
\end{tabular}




\section{Total Temperature}

Target Test Conditions: 130 knots, TTDC $=-1^{\circ} \mathrm{C}$
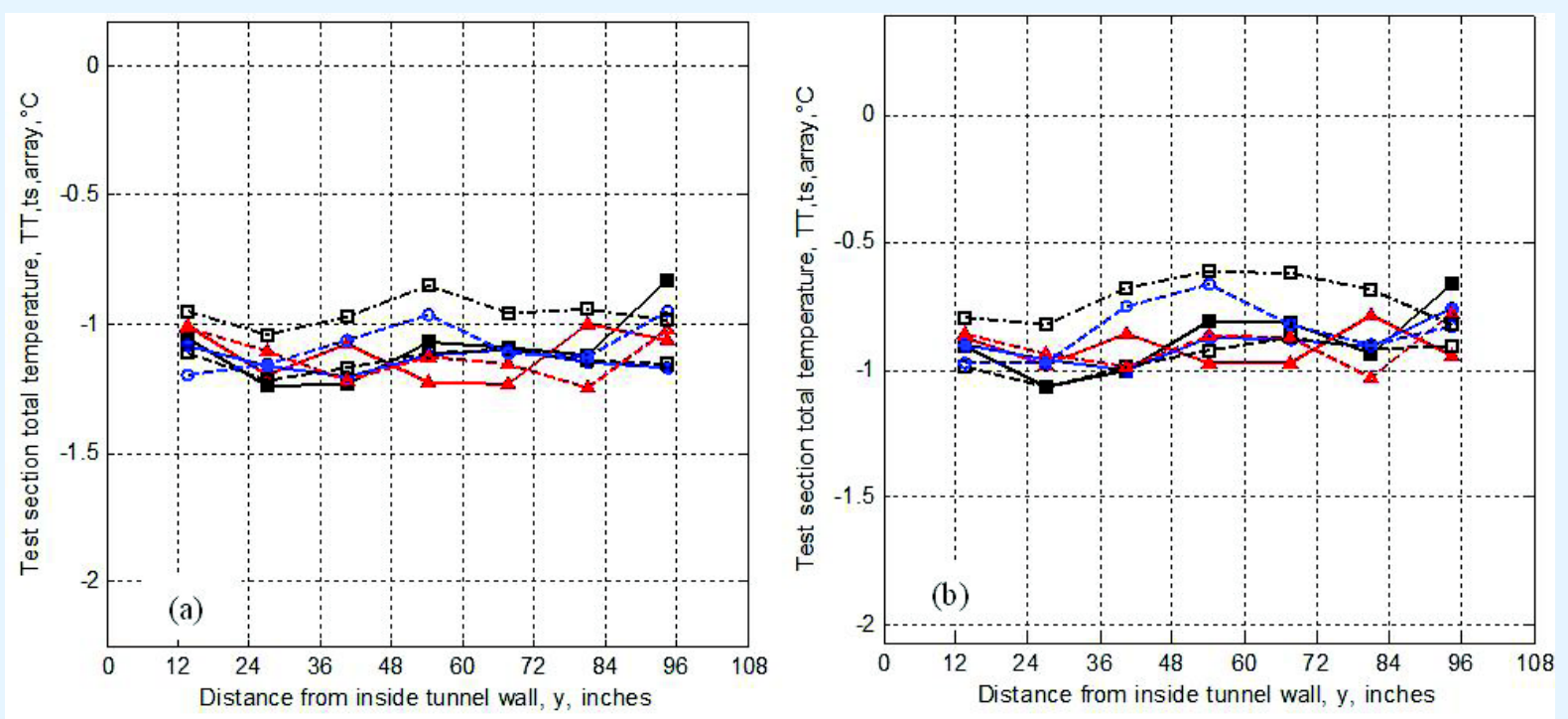

(a) 0 psig

(b) 30 psig

(c) 60 psig

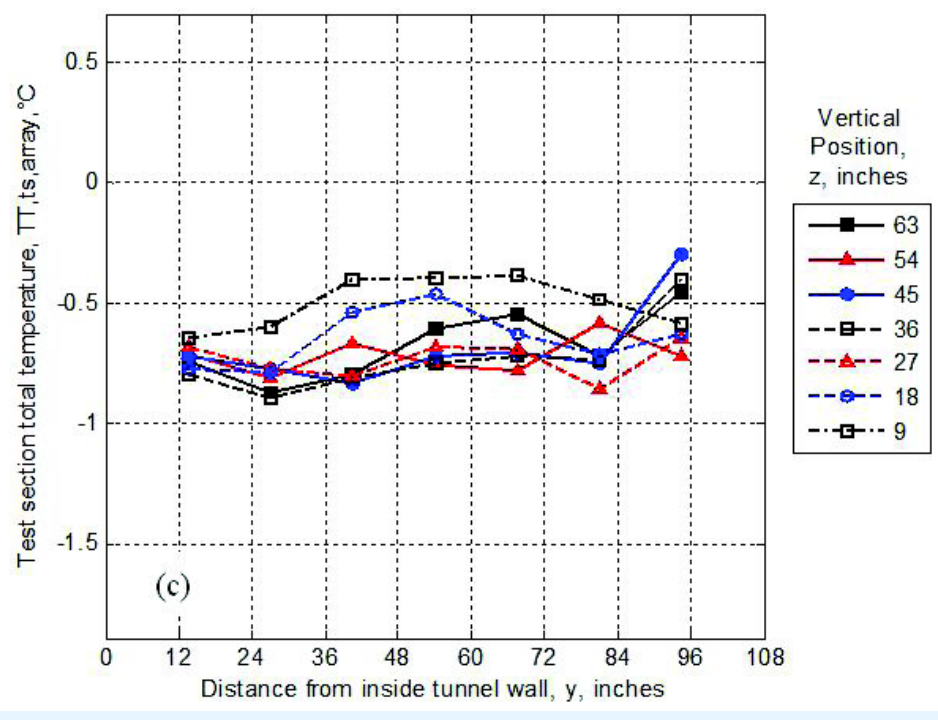




\section{Turbulence Intensity}

\section{9}

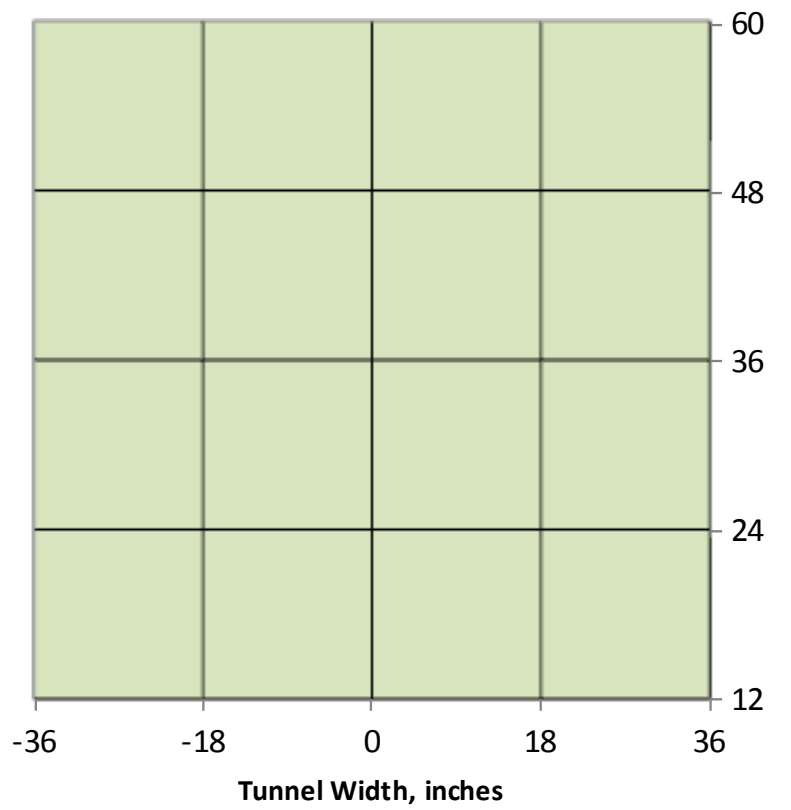

2012

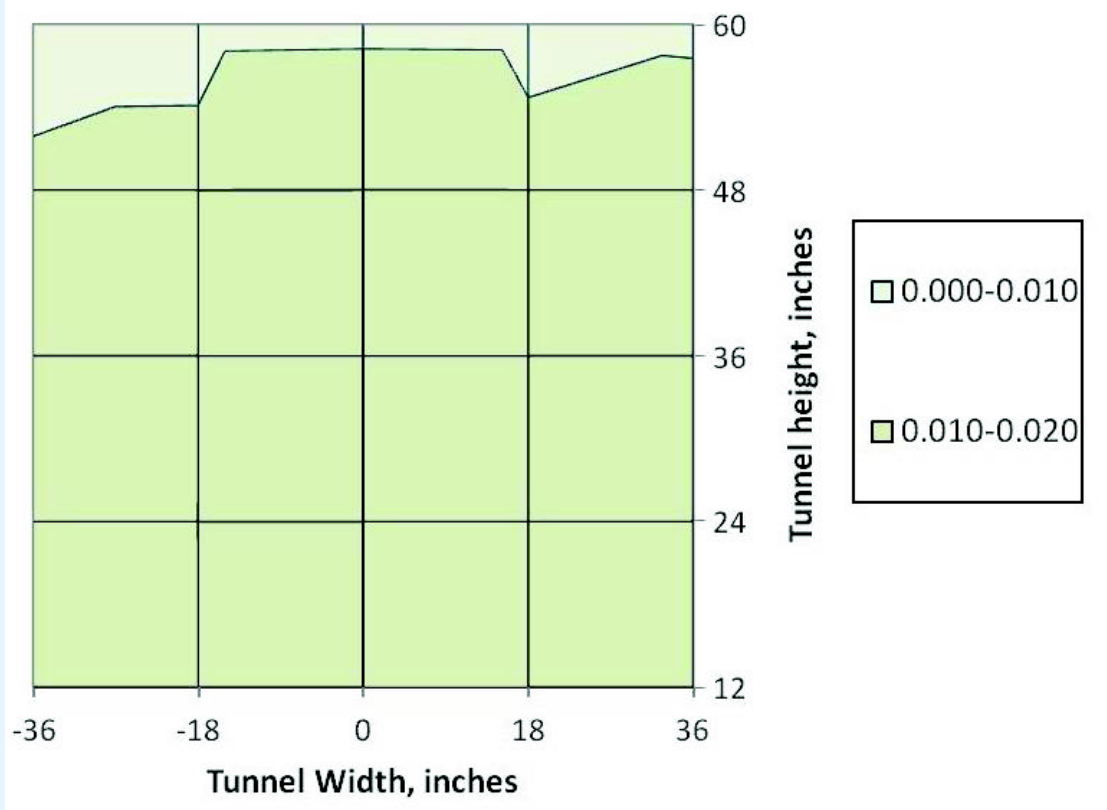

Target Test

Conditions:

150 knots,

Pair $=0$ psig 


\section{Mach number Calibration Curve}

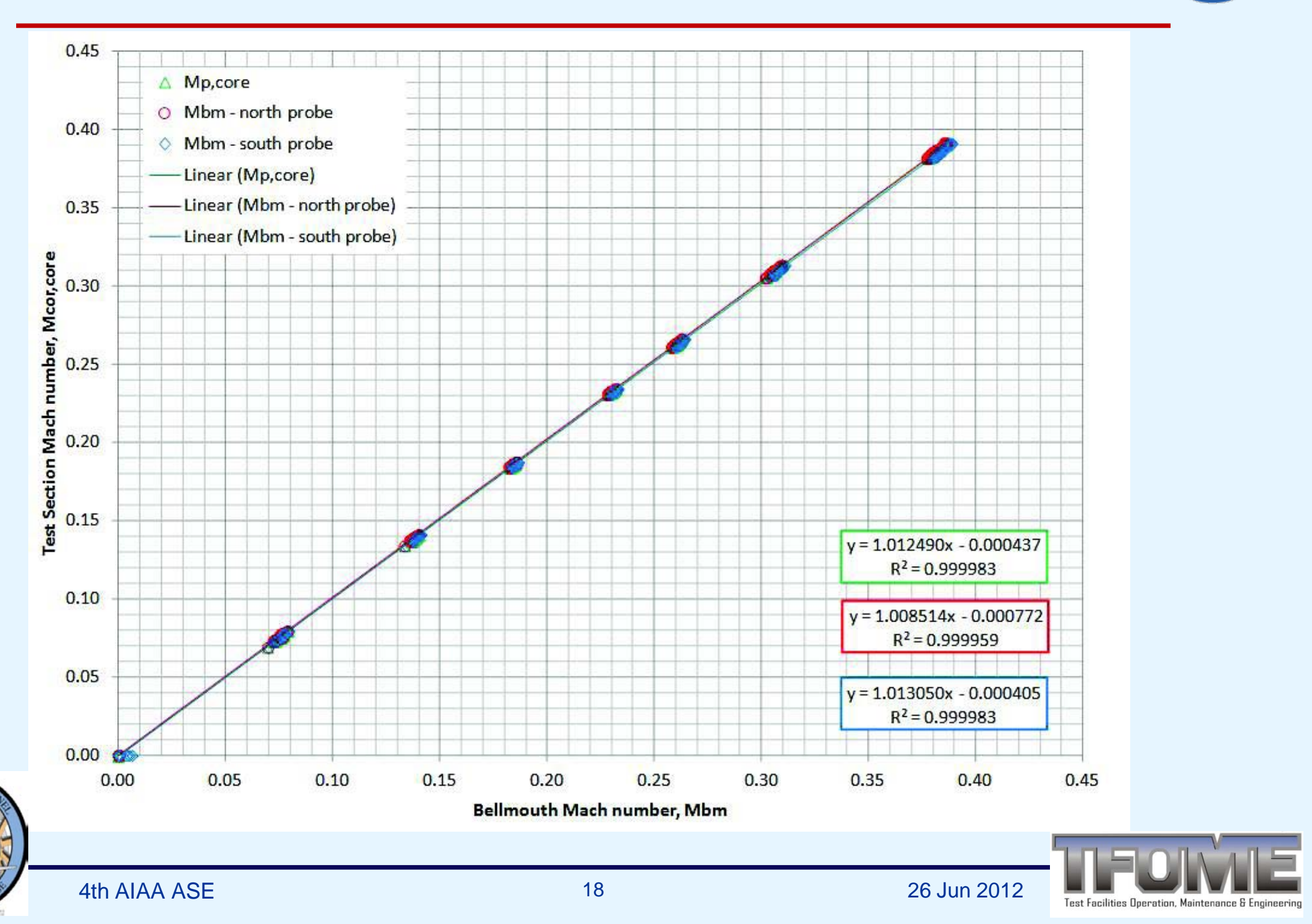




\section{Static Pressure Calibration Curve}

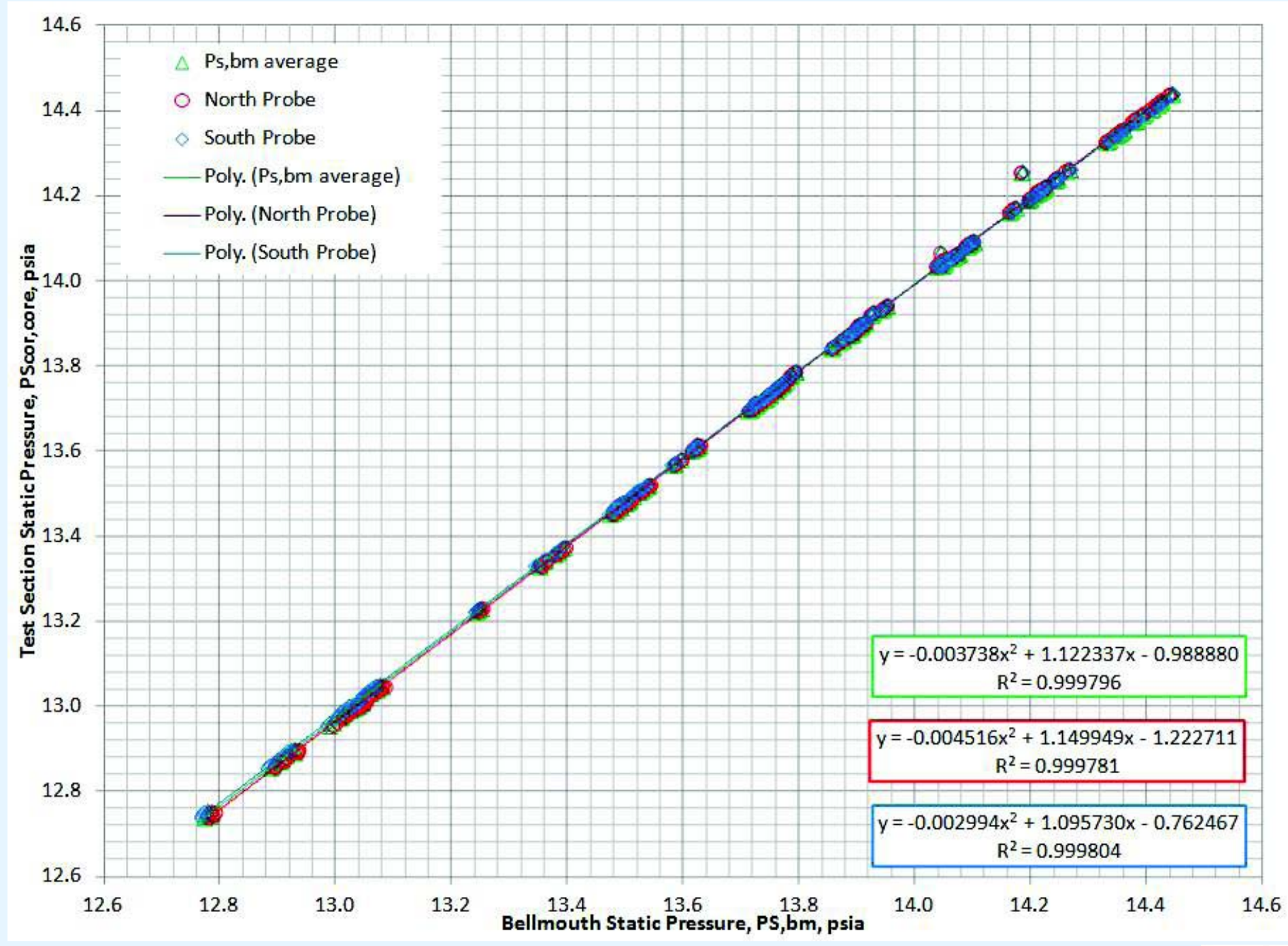




\section{Total Temperature Calibration Curve}

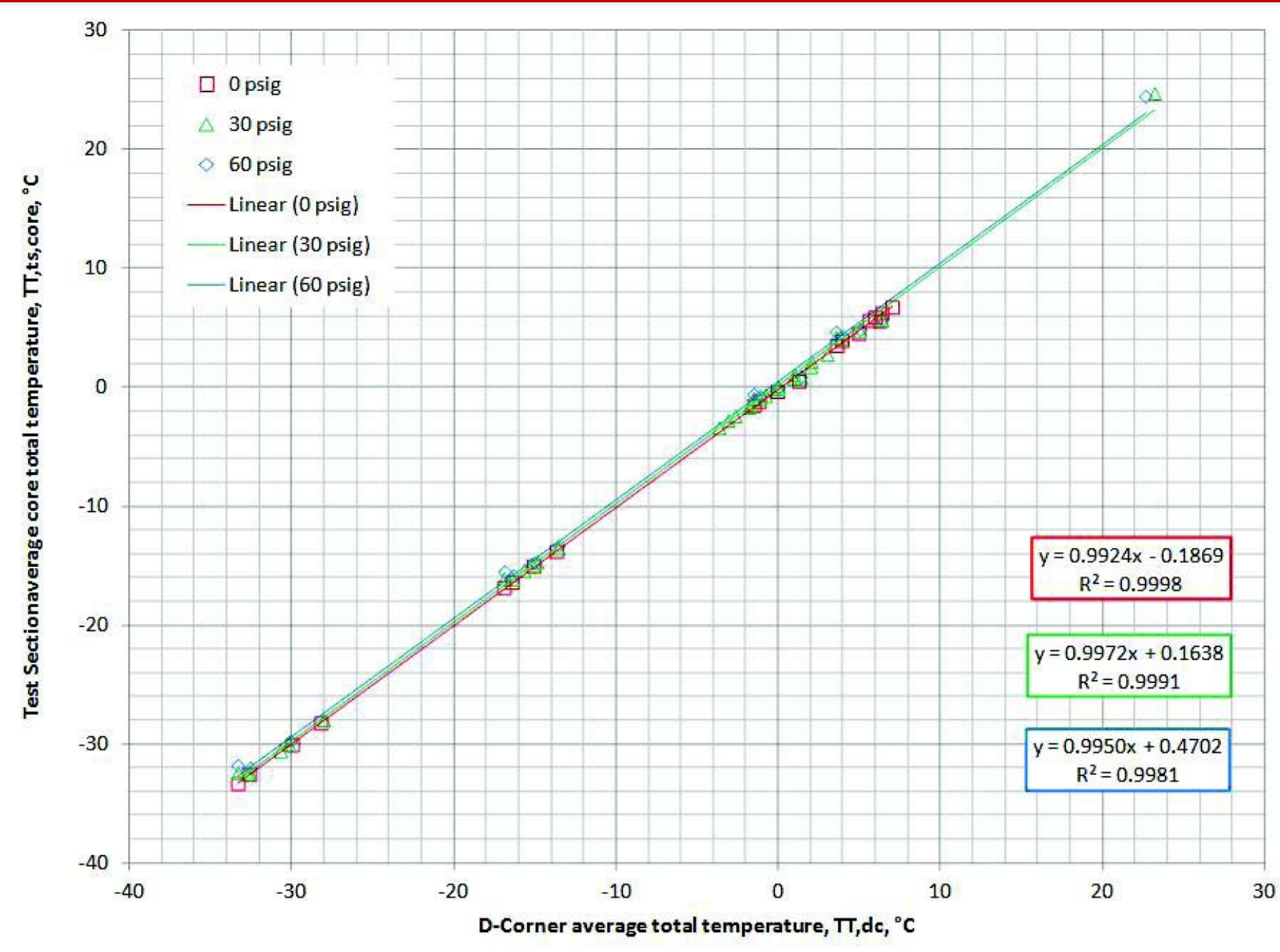




\section{Summary}

- Improvement in spatial temperature deviation of the heat exchanger

- New set of calibration curves have been implemented for static pressure, Mach number, and total temperature

- A facility health issue was resolved

- NASA CR will be out shortly with the full set of data. 


\section{IRT 2011-12 Cooling System Upgrade}

\section{Questions?}




\section{IRT 2011-12 Cooling System Upgrade}

\section{Backup Slides}




\section{No Struts vs. With Struts}

No Struts

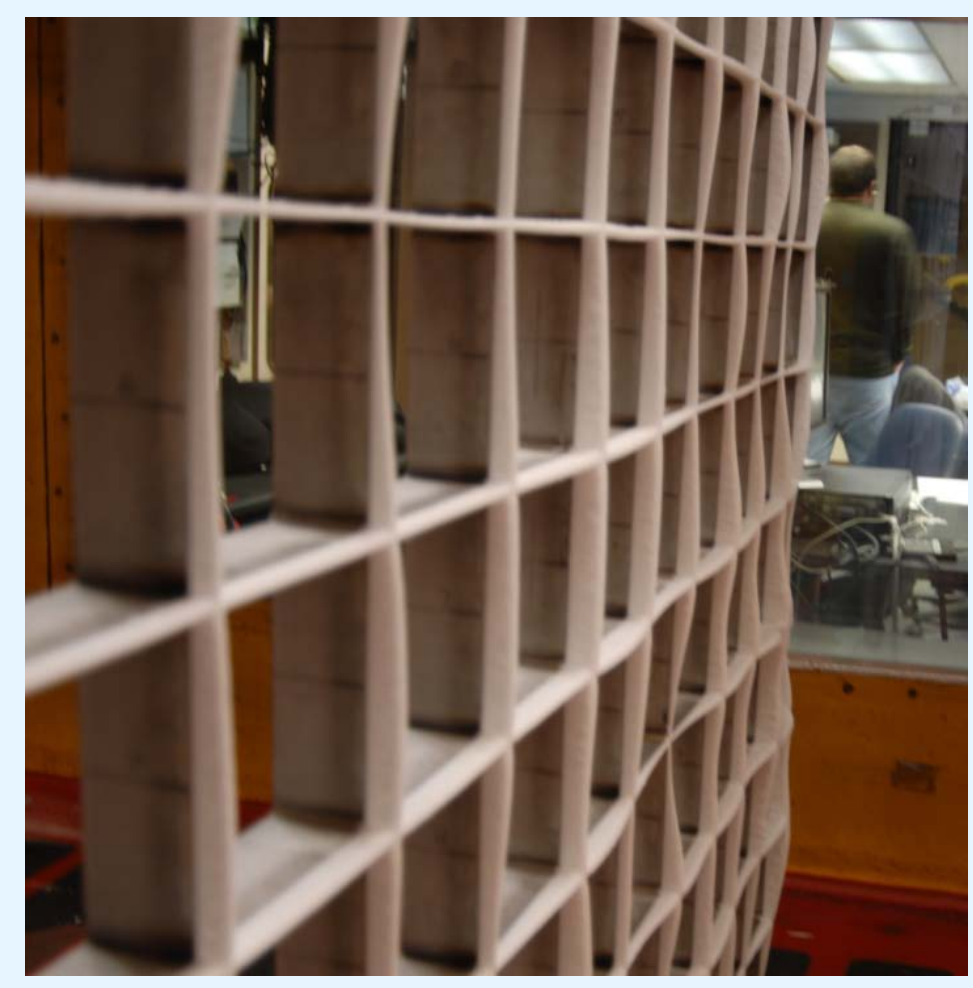

With Struts

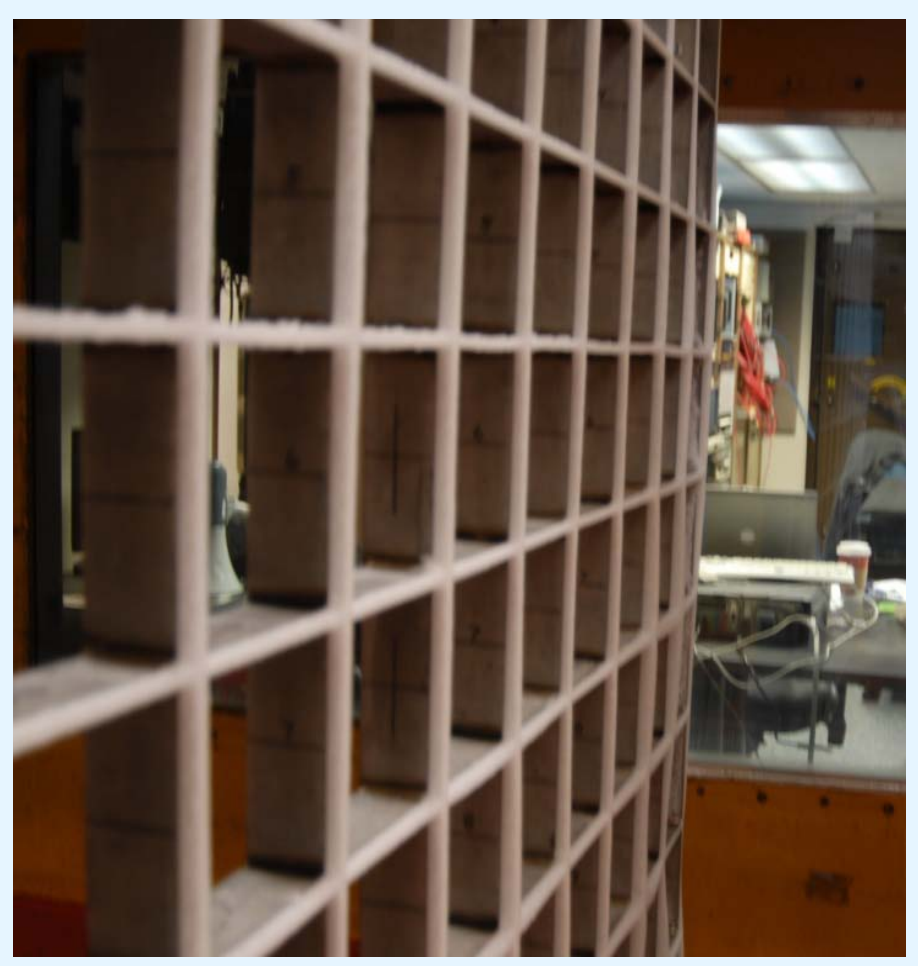




\section{5-hole pressure probe detail}

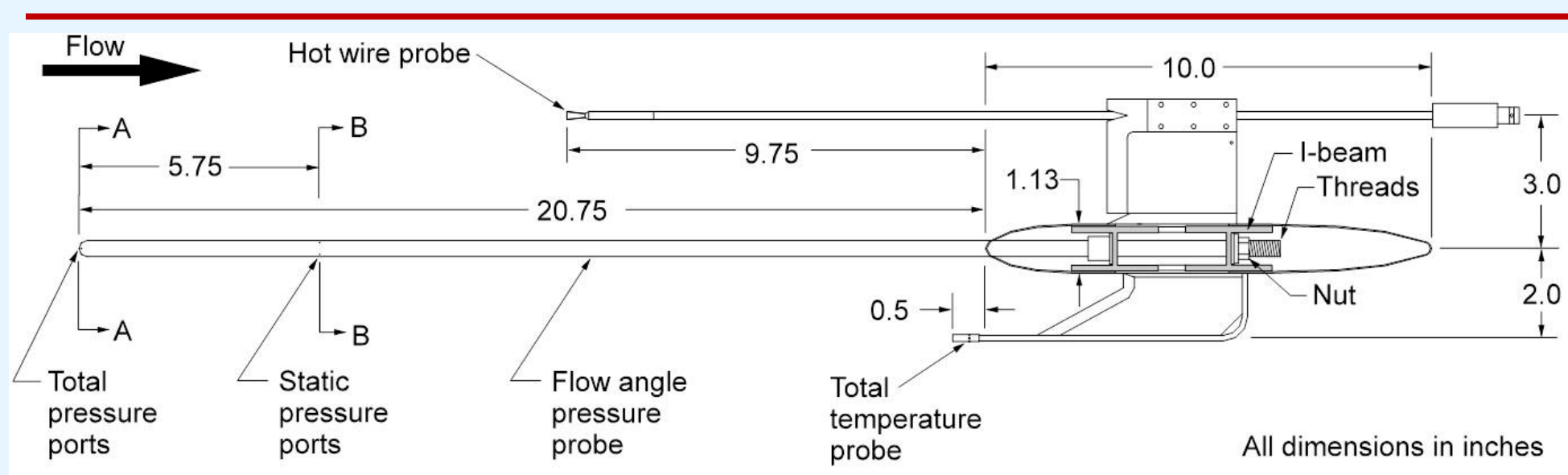

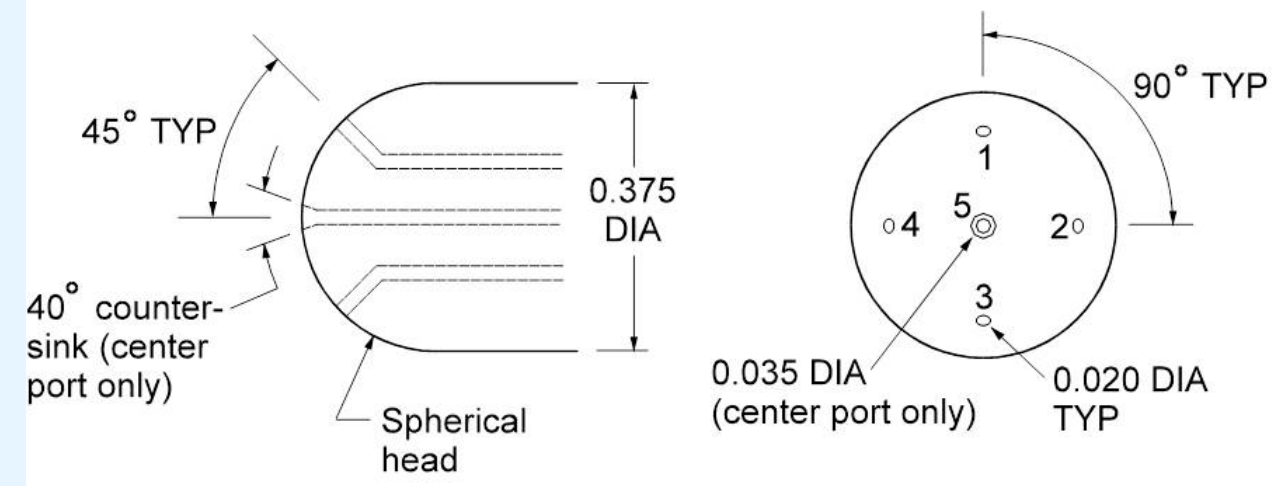

A-A

exploded

view

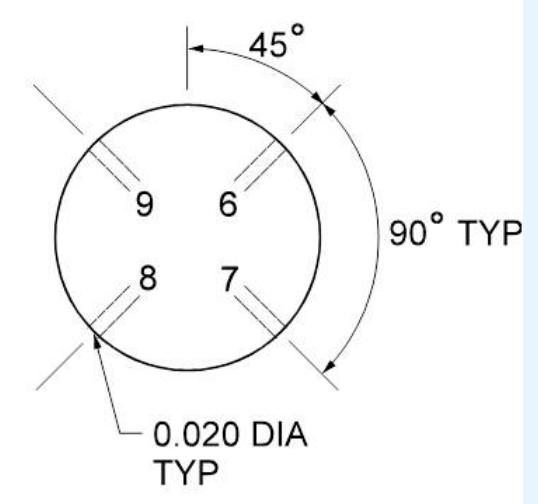

B-B

exploded

view

Total pressure port detail

Static pressure port detail 


\section{Turbulence Intensity (150 knots, 30psig)}

2012 Vel Uniformity: HWrake, V = 150, Pair = 30

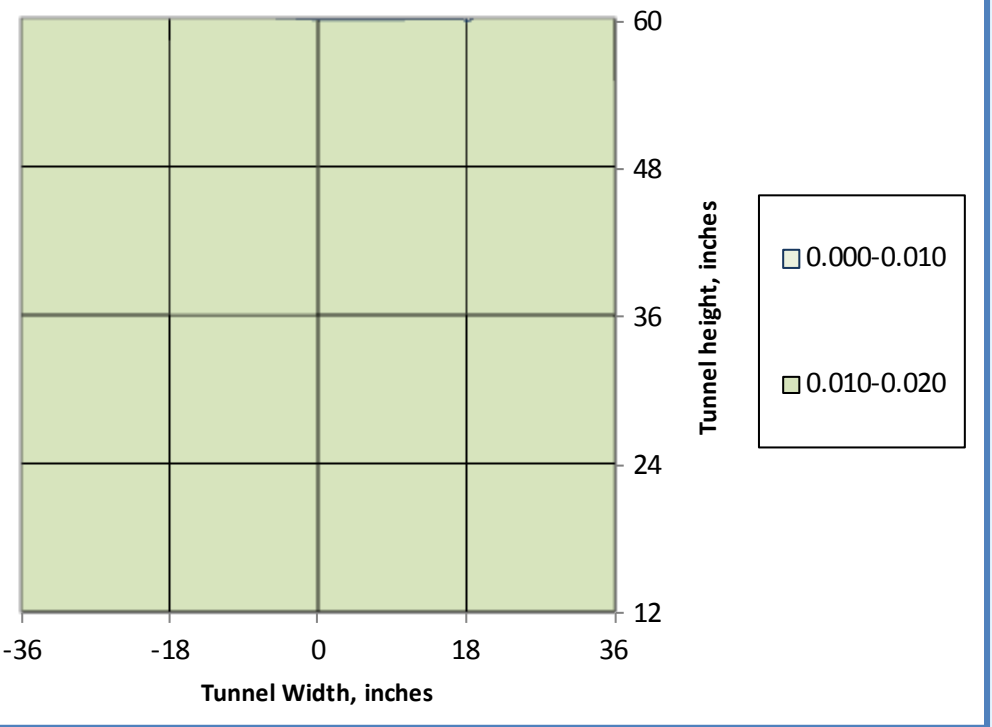

2009 Vel Uniformity: HWrake, V = 150, Pair = 30

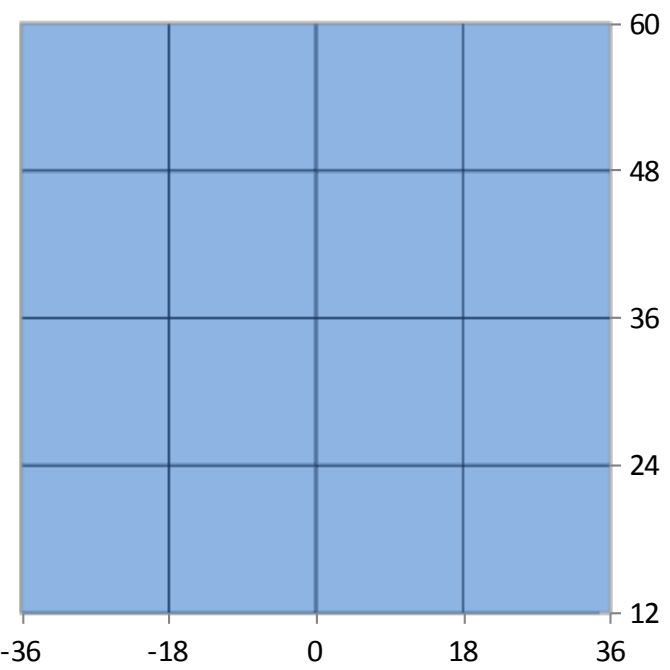

$\square 0.97-0.98$

$\square 0.98-0.99$

$\square 0.99-1.00$

$\square 1.00-1.01$

$\square 1.01-1.02$

$\square 1.02-1.03$ 


\section{Turbulence Intensity (150 knots, 60psig)}

2012 Vel Uniformity: HWrake, V = 150, Pair $=60$

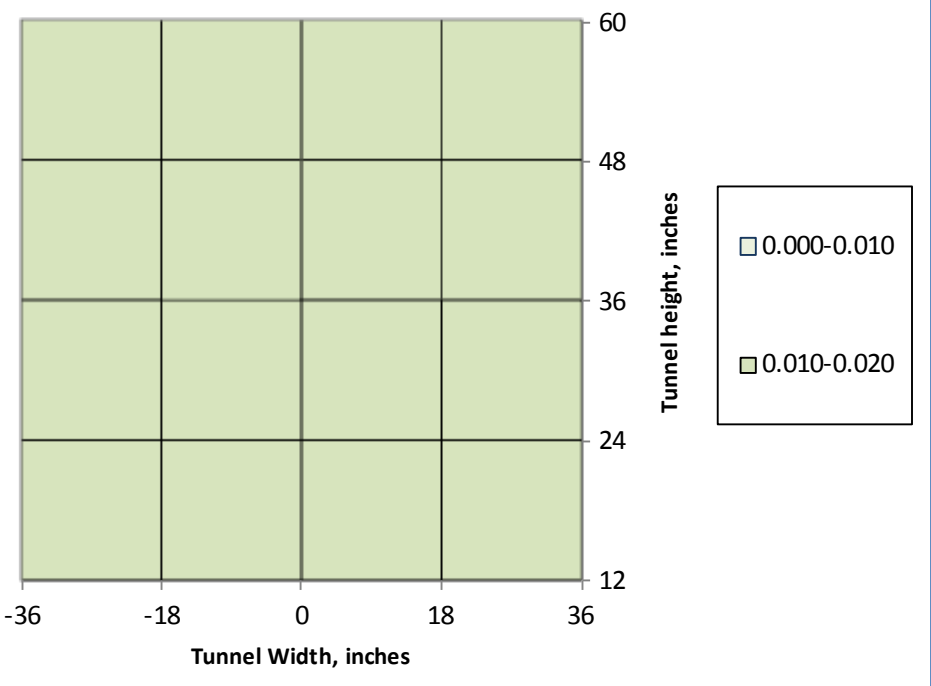

2009 Vel Uniformity: HWrake, V = 150, Pair $=60$

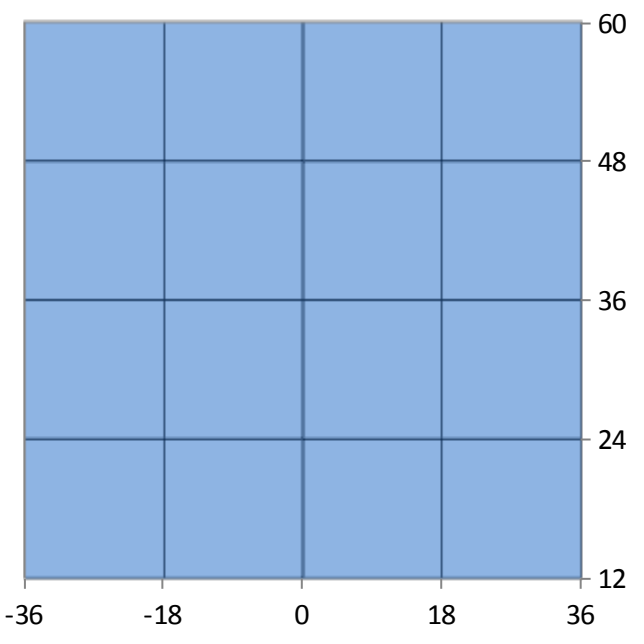

$\square 0.97-0.98$

$\square$ 0.98-0.99

$\square 0.99-1.00$

ㅁ. $1.00-1.01$

$\square 1.01-1.02$

$\square 1.02-1.03$ 


\section{Turbulence Intensity Historical Comparison}

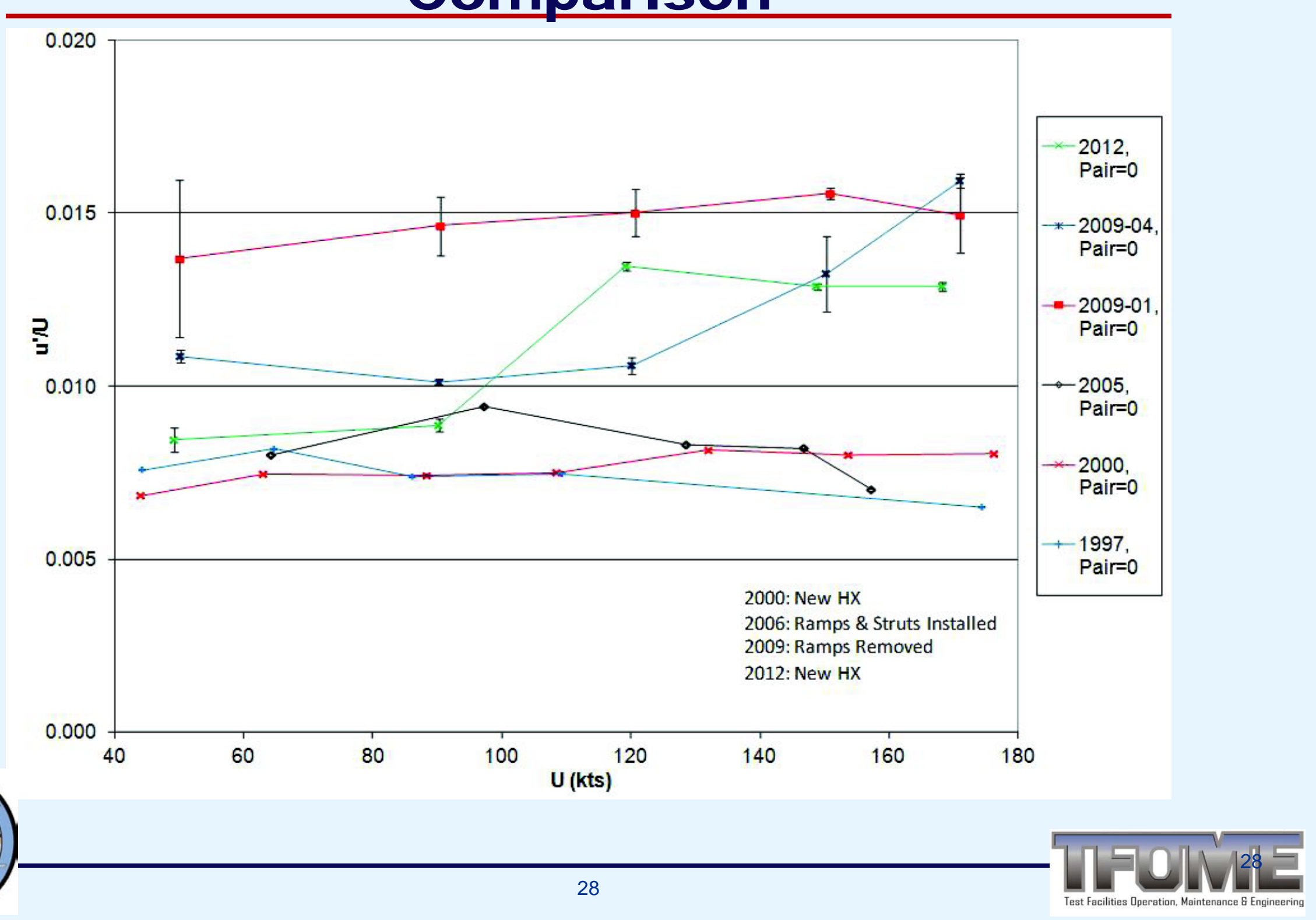

